\title{
KINEMATICS AND METALLICITIES IN THE BOÖTES III STELLAR OVERDENSITY: A DISRUPTED DWARF GALAXY?*
}

\author{
Jeffrey L. Carlin ${ }^{1}$, Carl J. Grillmair ${ }^{2}$, Ricardo R. Muñoz ${ }^{3}$, David L. Nidever ${ }^{1}$, and Steven R. Majewski ${ }^{1}$ \\ ${ }^{1}$ Department of Astronomy, University of Virginia, P.O. Box 400325, Charlottesville, VA 22904-4325, USA; jcarlin@virginia.edu, dln5q@virginia.edu, \\ srm4n@virginia.edu \\ ${ }^{2}$ Spitzer Science Center, 1200 East California Boulevard, Pasadena, CA 91125, USA; carl@ipac.caltech.edu \\ ${ }^{3}$ Yale University, Astronomy Department, P.O. Box 208101, New Haven, CT 06520-8101, USA; ricardo.munoz@yale.edu \\ Received 2009 June 12; accepted 2009 July 24; published 2009 August 11
}

\begin{abstract}
We report the results of a spectroscopic study of the Boötes III (BooIII) stellar overdensity carried out with the Hectospec multifiber spectrograph on the MMT telescope. Radial velocities have been measured for 193 BooIII candidate stars selected to have magnitudes and colors consistent with its upper main sequence and lower red giant branch, as well as a number of horizontal-branch candidates. From 20 identified candidate BooIII members, we measure a systemic velocity of $V_{\odot}=197.5 \pm 3.8 \mathrm{~km} \mathrm{~s}^{-1}$ and a velocity dispersion of $\sigma_{\mathrm{o}}=14.0 \pm 3.2 \mathrm{~km} \mathrm{~s}^{-1}$. We use the somewhat large velocity dispersion and the implied highly radial orbit, along with morphological evidence from Grillmair and stellar abundances, to argue that BooIII is likely the first known object observed in a transitional state between being a bound dwarf galaxy and a completely unbound tidal stream.
\end{abstract}

Key words: galaxies: individual (Boötes)

\section{INTRODUCTION}

Over the last few years and thanks to the advent of large-area photometric surveys, such as the Two Micron All Sky Survey (2MASS) and the Sloan Digital Sky Survey (SDSS; Abazajian et al. 2009), the substructured nature of the Milky Way halo has been clearly revealed. In recent years the number of known Galactic dwarf spheroidals (dSphs) has more than doubled (e.g., Willman et al. 2005; Belokurov et al. 2006a, 2007; Zucker et al. 2006a, 2006b; Grillmair 2006a), and numerous stellar streams have been identified (e.g., Ibata et al. 2001; Majewski et al. 2003; Rocha-Pinto et al. 2004; Yanny et al. 2003; Belokurov et al. 2006b; Grillmair 2006a, 2006b, 2009; Grillmair \& Dionatos 2006). These discoveries lend significant support to currently favored cold dark matter cosmological models, which predict that spiral galaxies form hierarchically from the merger and accretion of smaller subsystems (e.g., Bullock et al. 2001; Bullock \& Johnston 2005).

One exciting aspect of these discoveries is that they have revealed a previously unknown population of "ultrafaint" dwarf galaxies which are claimed to contain the highest dark matter fractions reported to date, with mass-to-light ratios above $1000(M / L)_{\odot}$ in some cases (e.g., Muñoz et al. 2006; Simon $\&$ Geha 2007; Strigari et al. 2008), but luminosities as low as $L_{V} \sim 300 L_{\odot}$ (Martin et al. 2007). A few of these satellites, Coma, Segue 1, Segue 2, and Boötes II, have properties closer to those of globular clusters than those of "classical" dSphs (e.g., half-light radii between 30 and $70 \mathrm{pc}$ ), and have been suggested to be satellites of satellites (Belokurov et al. 2009).

In this Letter, we present the results of a spectroscopic survey of another potential dwarf satellite, the Boötes III stellar overdensity (BooIII; Grillmair 2009, hereafter G09). This peculiar object, located at a distance of $46 \mathrm{kpc}$ and with $V$-band absolute magnitude of $M_{\mathrm{V}}=-5.8 \pm 0.5$ (Correnti et al. 2009), has a roughly power-law surface brightness profile and an irregular shape. Our study suggests that BooIII is likely a dwarf satellite in

\footnotetext{
* Observations reported here were obtained at the MMT Observatory, a joint facility of the Smithsonian Institution and the University of Arizona.
}

a transitional state between a bound entity and an unbound stellar stream. In this context, BooIII resembles the objects described by Kroupa (1997) but likely with a high dark matter content.

\section{SPECTROSCOPIC OBSERVATIONS AND DATA REDUCTION}

Radial velocities (RVs) were measured from spectra obtained in 2009 February with the Hectospec multifiber spectrograph (Fabricant et al. 2005) mounted at the $\mathrm{f} / 5$ focus on the $6.5 \mathrm{~m}$ MMT. Targets were selected from the SDSS stellar database to lie on or near the BooIII color-magnitude sequence from G09, in a magnitude range of $18.5<g<22.5$. We also included candidate blue horizontal-branch (BHB) stars that are near the BHB locus in Figure 13 of G09, bringing the total number of assigned targets in the $\sim 1$ degree Hectospec field of view to 227. Figure 1 shows the $g-i$ versus $g$ color-magnitude diagram (CMD) for all stars within 0.5 of the BooIII center, as well as an old, metal-poor isochrone chosen to be similar to that of M15 (the cluster which guided our target selection).

We used the $600 \mathrm{gpm}$ grating, centered at $5800 \AA$, to give a working wavelength range of 4550-7050 $\AA$ at a dispersion of $\sim 0.55 \AA$ pixel $^{-1}$ (2.1 $\AA$ resolution). This region was selected to include the $\mathrm{H} \beta, \mathrm{Mg}$ triplet, $\mathrm{Na} \mathrm{D}$, and $\mathrm{H} \alpha$ spectral features. Queue-observed integrations totaled $3 \times 1800 \mathrm{~s}$ on UT 2009 February 3, and $6 \times 1800 \mathrm{~s}$ on UT 2009 February 20, for a summed exposure of $4.5 \mathrm{hr}$ on this field. Sky fibers (57 in all) were assigned throughout the field, distributed so that a number of them would fall within each of the two CCD chips of the Hectospec system. A total of 193 target spectra had starlike spectra (there were a number of background quasars), with sufficient signal-to-noise ratio $(\mathrm{S} / \mathrm{N}>10)$ for $\mathrm{RV}$ measurement.

The Hectospec data were reduced using an external version of the SAO "SPECROAD" reduction pipeline (Mink et al. 2007) written by Juan Cabanela, called ESPECROAD. ${ }^{4}$ The pipeline automates many reduction steps, including bias-correction, flat-fielding, cosmic-ray rejection, fiber-to-fiber throughput

\footnotetext{
4 http://iparrizar.mnstate.edu/ juan/research/ESPECROAD/index.php
} 


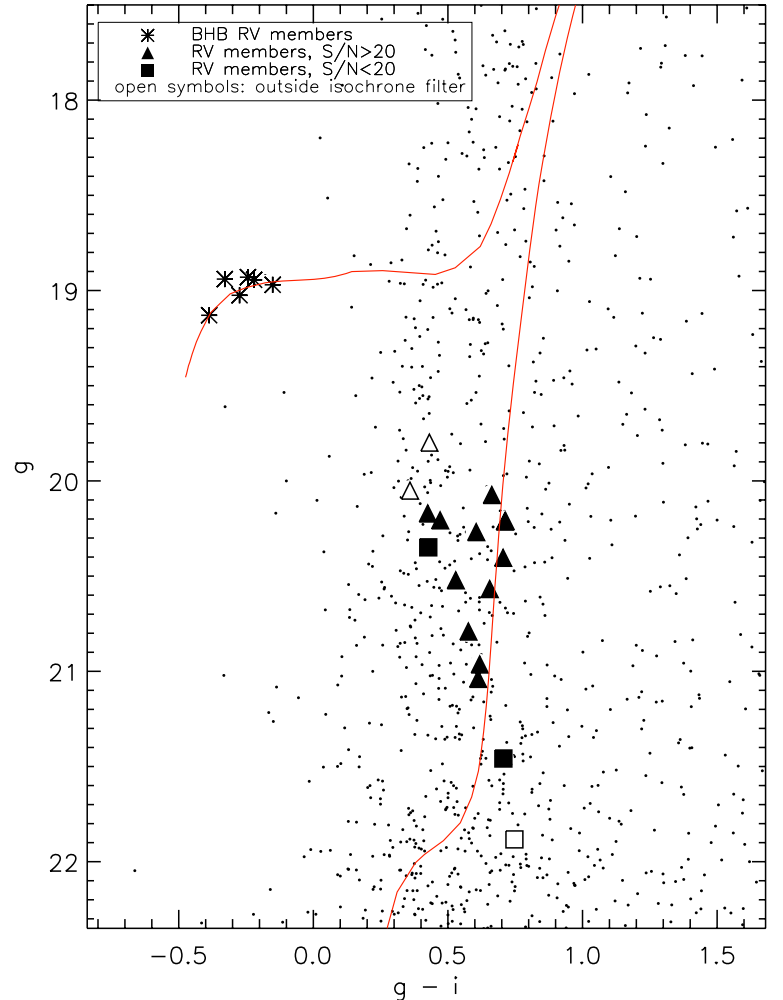

Figure 1. SDSS CMD of a 30 arcmin radius about the nominal BooIII center, drawn from the same data points as Figure 13 of Grillmair (2009). The solid line is an old, metal-poor $([\mathrm{Fe} / \mathrm{H}]=-2.30)$ isochrone from Girardi et al. (2000), shifted to a distance of $46.0 \mathrm{kpc}$. Large symbols represent all stars within the $3 \sigma \mathrm{RV}$ selection centered at $\sim 197.5 \mathrm{~km} \mathrm{~s}^{-1}$; asterisks represent the six BHB candidates that were in our spectroscopic sample, triangles are RV members whose spectra had $\mathrm{S} / \mathrm{N}>20$, and squares those having $\mathrm{S} / \mathrm{N}<20$. Filled symbols and asterisks are candidate RV members that are also within $0.25 \mathrm{mag}$ of the metal-poor isochrone in the CMD; these constitute our final BooIII sample.

adjustments, and sky subtraction. Wavelength calibration was performed manually using sets of three combined HeNeAr calibration lamp exposures from each night of observing.

We derived RVs using the IRAF task FXCOR to crosscorrelate object spectra against two template G8V RV standards, each observed three times. We first correlated the standard spectra against each other, and found that to achieve agreement between our measurements and published $\mathrm{RVs}^{5}$ for these stars, it was necessary to shift all RVs measured against one of them by $6 \mathrm{~km} \mathrm{~s}^{-1}$. After doing so, the measured RVs for all six standard spectra agree with published values to within $1.0 \mathrm{~km} \mathrm{~s}^{-1}$. To minimize the effects of noise for spectra with lower $\mathrm{S} / \mathrm{N}$, the cross-correlation was restricted to the regions around the $\mathrm{H} \alpha$, $\mathrm{Mg}$ triplet, and $\mathrm{H} \beta$ lines. The standard deviation of six RV measurements for most objects (after applying the $6 \mathrm{~km} \mathrm{~s}^{-1}$ shift discussed above) was less than $1 \mathrm{~km} \mathrm{~s}^{-1}$, increasing to $\sim 3 \mathrm{~km} \mathrm{~s}^{-1}$ at $g \sim 22$.

To estimate RV uncertainties, we separated our nine individual object exposures into three sets of three summed exposures, totaling $1.5 \mathrm{hr}$ of exposure each. We then measured RVs in each of these three independent frames, and fit a curve to the run of standard deviation of the three measurements as a function of magnitude. We used this fit to the trend of $\sigma_{\mathrm{RV}}$ versus $g$ for the brighter stars in our sample to assign an RV uncertainty for

\footnotetext{
5 From the Geneva Radial Velocity Standard Stars at http://obswww.unige.ch/ $\sim$ udry $/ \mathrm{std} /$
}

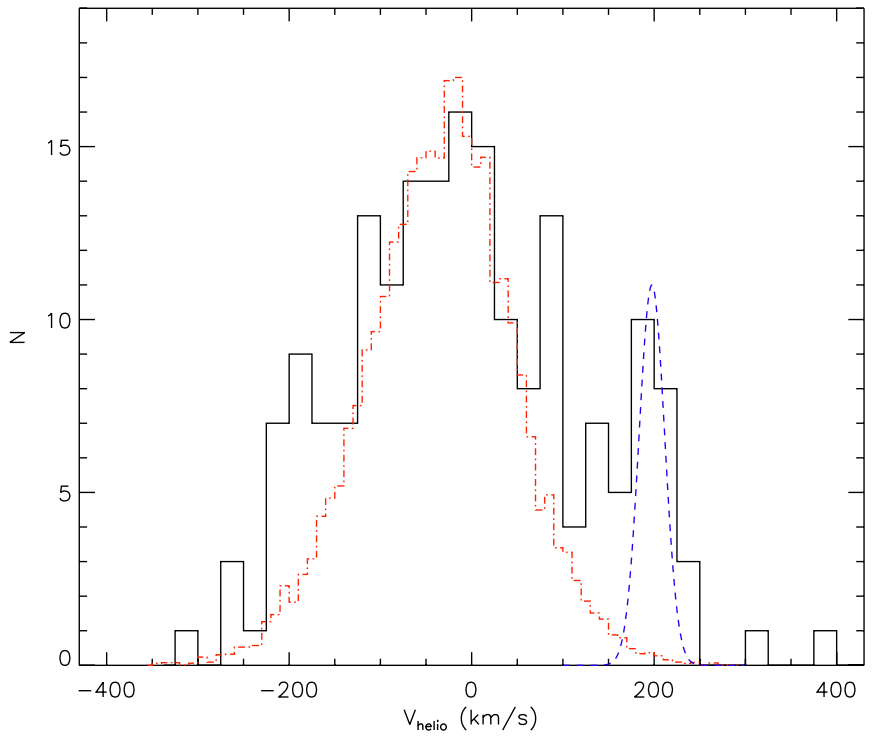

Figure 2. Heliocentric RVs (solid histogram) for all stars in the MMT+Hectospec configuration with reliable measurements. For comparison, we show scaled output from the Besançon model as the red dot-dashed histogram. The model data consist of stars within $0.2<g-r<0.6$, and 18.6 $<g<22.0$, which are predominantly Milky Way halo turnoff stars in the same region of the CMD as our targets. Relative to the model, there are two peaks of "excess" counts: one at roughly $-180 \mathrm{~km} \mathrm{~s}^{-1}$, and a second at $V \sim 200 \mathrm{~km} \mathrm{~s}^{-1}$. We identify this latter peak as associated with BooIII. The blue dashed curve is a Gaussian with mean velocity $V_{\odot}=197.5 \mathrm{~km} \mathrm{~s}^{-1}$ and a width of $\sigma_{\mathrm{o}}=$ $14.0 \mathrm{~km} \mathrm{~s}^{-1}$, matching our final derived values for BooIII.

each star we observed based on its magnitude (extrapolating the curve for fainter stars having insufficient signal to measure an $\mathrm{RV}$ from the $1.5 \mathrm{hr}$ exposures). Velocity uncertainties vary from $\sigma_{\text {Vhelio }} \sim 3 \mathrm{~km} \mathrm{~s}^{-1}$ at $g=18.5$ to $\sim 10 \mathrm{~km} \mathrm{~s}^{-1}$ at $g=21$ (and higher at fainter magnitudes).

\section{SPECTROSCOPIC RESULTS}

\subsection{Boötes III Membership}

Figure 2 shows RVs for all stars in our sample with reliable measurements $(\mathrm{S} / \mathrm{N}>10)$. For comparison, we show (red dotdashed curve) a scaled RV distribution from $\sim 100$ realizations of the Besançon Galactic model (Robin et al. 2003) along this line of sight, covering the same magnitude and color ranges as our targets. Since this field is at high Galactic latitude $(b \sim 75.4)$, blue stars at faint magnitudes are mostly Milky Way halo main-sequence turnoff stars, with a large $\left(\sim 100 \mathrm{~km} \mathrm{~s}^{-1}\right)$ velocity dispersion. This halo distribution is well matched by our data set. However, two significant excesses with respect to the expected halo distribution, at $V_{\text {helio }}$ of $-180 \mathrm{~km} \mathrm{~s}^{-1}$ and $\sim 200 \mathrm{~km} \mathrm{~s}^{-1}$, are visible in the histogram. To determine whether one of these RV signatures is associated with BooIII we check the velocities of the six candidate BooIII BHB stars we have included from the "clump" in Figure 13 of G09 (asterisk symbols in Figure 1). Interestingly, all six of these stars have velocities within the $\sim 200 \mathrm{~km} \mathrm{~s}^{-1}$ peak, with a mean value of $V_{\text {helio }}=195.6 \pm 7.2 \mathrm{~km} \mathrm{~s}^{-1}$ and dispersion $13.7 \mathrm{~km} \mathrm{~s}^{-1}$. Since none of the BHB candidates have velocities within the negative RV peak, we identify this positive, high-velocity peak with the BooIII kinematical signature.

Having thus identified BooIII's systemic velocity, we select other stars within $3 \sigma$ of this BHB velocity peak as candidate BooIII members. These stars are highlighted in the $g-i$ versus $g$ CMD in Figure 1. The good agreement between the isochrone 


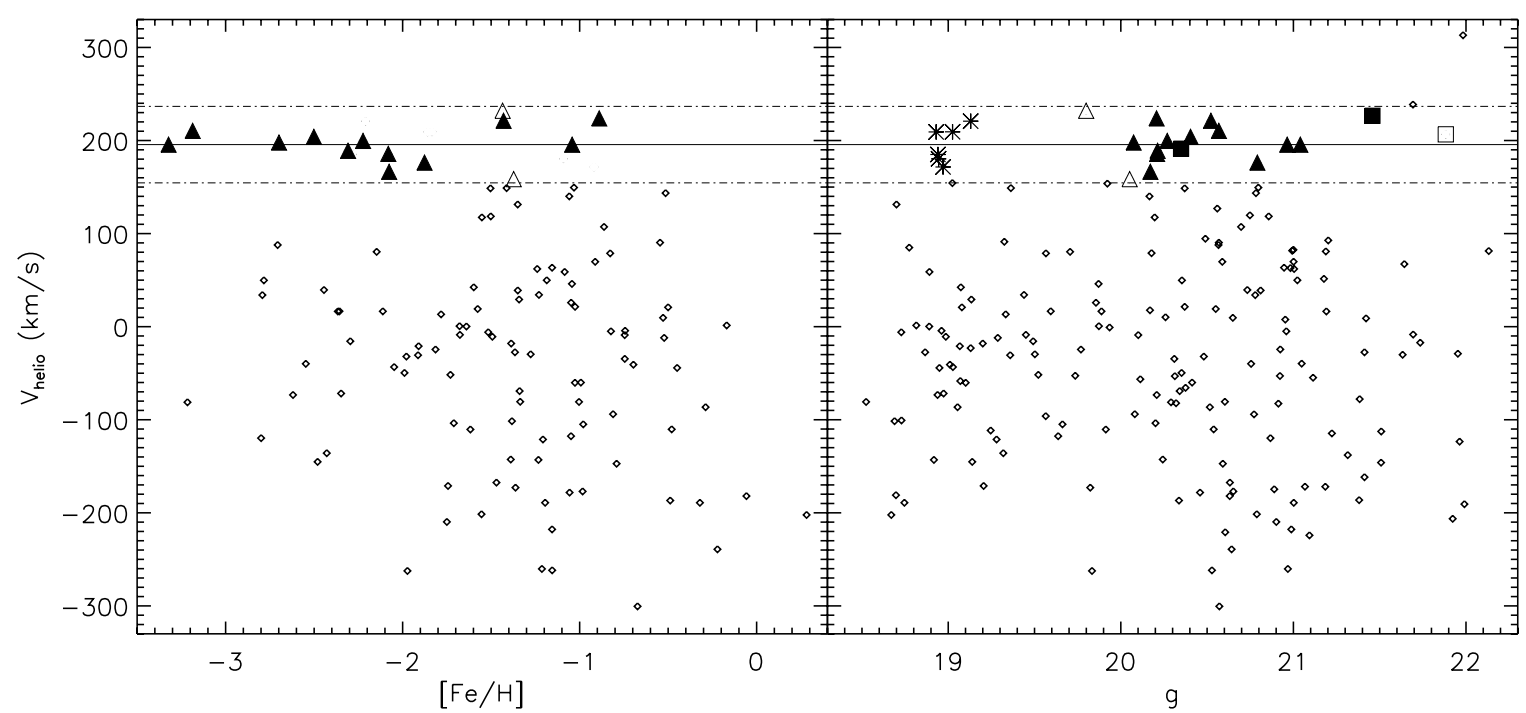

Figure 3. Left panel: heliocentric RVs as a function of metallicity ([Fe/H]) for all stars in our observed sample having spectra with $\mathrm{S} / \mathrm{N}>20$. Right panel: RVs vs. SDSS $g$ magnitude for all stars with spectra. Symbols for both panels are as in Figure 1. As in previous plots, open symbols are those stars that were excluded from the final sample of BooIII candidates. The horizontal lines in both panels represent the mean (solid line) and $3 \sigma$ (dot-dashed line) RV selection criteria based on the BHB stars.

for an old, metal-poor $([\mathrm{Fe} / \mathrm{H}]=-2.30)$ population and the BooIII RV candidates suggests that we are indeed seeing the red giant branch of an old, metal-poor, comoving population of stars.

Cross-correlation of BHB spectra in our sample against the two available G-dwarf standard spectra results in RVs with higher uncertainties than we derive for the later-type stars in our sample. This is due to the mismatch in spectral type and spectral line profiles between the BHB candidates and the RV standard stars. Because of this, the scatter in our BHB RVs is likely artificially high, potentially leading to higher field-star contamination in our $3 \sigma$ velocity selection. In order to reach a cleaner sample of BooIII members, we further exclude stars that are more than 0.25 mag from the isochrone shown in Figure 1. This leaves our final sample of 20 BooIII candidate members (14 if the BHB stars are excluded), which is represented in Figures 1, 3, and 4 with large filled symbols and asterisks.

\subsection{Metallicity}

Stellar atmospheric parameters for all observed stars other than the BHB candidates are determined using low-resolution Lick spectral indices (as defined by Worthey et al. 1994). Measured indices for all input spectra are placed onto the standard Lick index system of Schiavon (2007) using offsets derived from standard spectra observed with the same telescope/ instrument setup. We derive a suitable estimate of the effective temperature, $T_{\text {eff }}$, of each star based on its $(g-r)$ photometric color. Relatively accurate dwarf/giant separation is then performed using the gravity-sensitive Mg b feature at $\sim 5170 \AA$, with stars sorting well by gravity in the $\mathrm{Mg} \mathrm{b}-\mathrm{H} \beta$ index plane $\left(\mathrm{H} \beta\right.$ is primarily sensitive to $\left.T_{\text {eff }}\right)$. The metallicity, $[\mathrm{Fe} / \mathrm{H}]$, for each star is determined using two methods; from the Schiavon (2007) spectral library we have derived separate polynomial surface fits of $(1)[\mathrm{Fe} / \mathrm{H}]$ to the Lick iron indices and stellar color and $(2)[\mathrm{Fe} / \mathrm{H}]$ as a function of the iron indices and $\mathrm{H} \beta$ (similar to the Friel (1987) interpolation between lines of constant metallicity, or the Gorgas et al. (1993) fitting functions). Thus, moderately accurate estimates of $[\mathrm{Fe} / \mathrm{H}]$ derive from the combination of all six Lick iron indices falling within the ob-

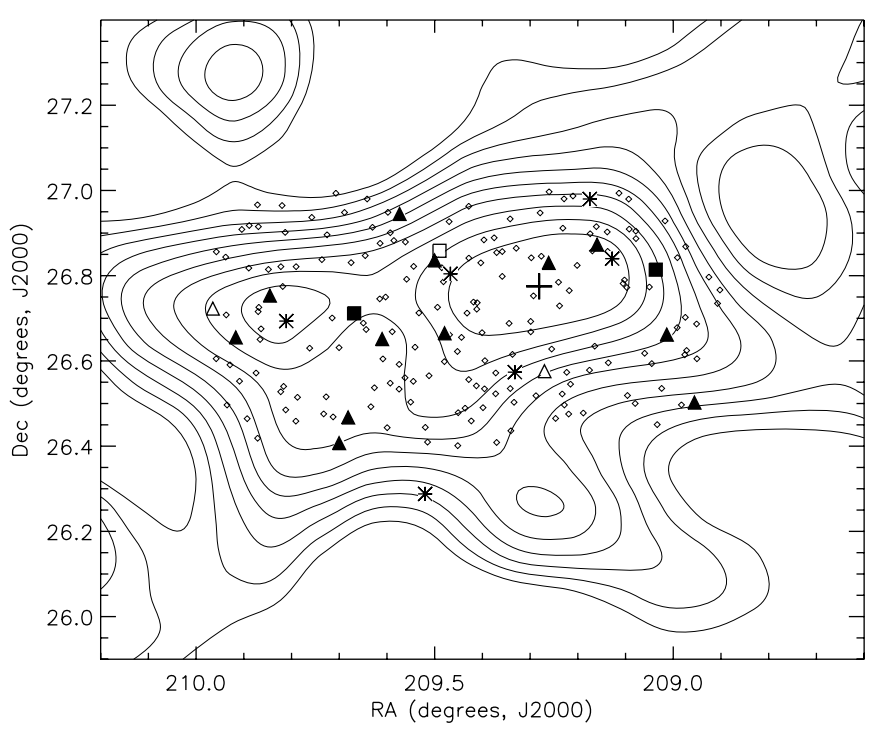

Figure 4. Spatial distribution of all observed spectroscopic targets. Symbols as in Figure 3. Contours represent filtered star counts, and the large plus sign denotes the BooIII center, both from the G09 study.

served spectral bandpass as well as the stellar color and $\mathrm{H} \beta$ index.

Measured metallicities as a function of $V_{\text {helio }}$ are shown in Figure 3. We estimate typical uncertainties in $[\mathrm{Fe} / \mathrm{H}]$ of $\sim 0.3-$ $0.5 \mathrm{dex}$, depending on the magnitude of the star. According to Grillmair (2009), the matched-filter signal of BooIII is strongest for the most metal-poor filter applied $(\mathrm{M} 15:[\mathrm{Fe} / \mathrm{H}]=-2.26$; Harris 1996). Furthermore, the M15 ridgeline provides the best fit to the CMD locus of BooIII (G09, Figure 12). We thus expect the majority of BooIII members to be metal-poor ([Fe/ $\mathrm{H}] \lesssim-2.0)$. Examining the stars within the $3 \sigma \mathrm{RV}$ selection in Figure 3, we note that a number of candidates are indeed rather metal-poor. Furthermore, both of the stars with $\mathrm{S} / \mathrm{N}>20$ spectra that were excluded from the final sample as likely foreground contaminants (i.e., open triangles in Figures 1, 3, and 4) have measured $[\mathrm{Fe} / \mathrm{H}]>-1.8$. 
From the individual stellar metallicities of our final sample (excluding the BHB stars), we derive a maximum likelihood estimation of $[\mathrm{Fe} / \mathrm{H}] \approx-2.1 \pm 0.2$ for BooIII. Since these individual values are derived from low $\mathrm{S} / \mathrm{N}$ spectra, we shift the BooIII candidate spectra to rest wavelength and stack them to produce a higher $\mathrm{S} / \mathrm{N}$ spectrum. Doing so, we derive $[\mathrm{Fe} / \mathrm{H}] \approx-2.0$, which is consistent with all other measurements for BooIII. At the integrated $V$-band absolute magnitude of BooIII, $M_{\mathrm{V}}=-5.8 \pm 0.5$ (Correnti et al. 2009), our measured metallicity is typical for a dwarf galaxy (see Figure 11 of Simon $\&$ Geha 2007), and more metal-poor than all Galactic globular clusters at similar luminosity. As is obvious from Figure 3, there is a fairly large metallicity spread in the BooIII candidate stars $\left(\sigma_{[\mathrm{Fe} / \mathrm{H}]} \approx 0.6\right)$. This may be due to foreground contamination of our sample, though as seen in Figure 2, very few Galactic stars are expected at these velocities. This spread in $[\mathrm{Fe} / \mathrm{H}]$ is comparable to (though slightly higher than) those found by Simon \& Geha (2007) for a number of other ultrafaint dwarfs (see their Table 4), and may reflect the presence of multiple generations of star formation among the BooIII stellar population.

\subsection{Velocity Dispersion and Mass Estimate}

With our final sample of BooIII candidates, we use the maximum likelihood method (Pryor \& Meylan 1993; Hargreaves et al. 1994; Kleyna et al. 2002) to derive the systemic velocity and velocity dispersion of BooIII. For the full sample (20 stars, including the $6 \mathrm{BHB}$ stars), we find $V_{\odot}=197.5 \pm 3.8 \mathrm{~km} \mathrm{~s}^{-1}$ and a velocity dispersion of $\sigma_{\mathrm{o}}=14.0 \pm 3.2 \mathrm{~km} \mathrm{~s}^{-1}$. Removing the BHB stars (leaving 14 total), the result is virtually unchanged: $V_{\odot}=198.2 \pm 4.5 \mathrm{~km} \mathrm{~s}^{-1}$, and $\sigma_{\mathrm{o}}=$ $14.1 \pm 3.7 \mathrm{~km} \mathrm{~s}^{-1}$-we thus consider only the full sample henceforth. At the position and distance of BooIII, this $V_{\odot}$ converts to an unusually high Galactic standard of rest velocity of $V_{\mathrm{GSR}}=238.8 \pm 3.8 \mathrm{~km} \mathrm{~s}^{-1}$ which implies that BooIII is on a very radial (and thus potentially destructive) orbit. This idea of a high-latitude object on a rather eccentric orbit agrees with the suggestion by G09 that BooIII may be extended along the line of sight (i.e., along its orbit).

Our measured dispersion of $14 \mathrm{~km} \mathrm{~s}^{-1}$ for BooIII is the highest measured line-of-sight velocity dispersion of any dSph, even higher than the central dispersion of Sagittarius, the most massive of the Galactic dSph galaxies. It has been shown in previous studies (e.g., Piatek \& Pryor 1995; Oh et al. 1995; Kroupa 1997; Muñoz et al. 2008) that even if a satellite is experiencing tidal disruption, its $\sigma_{\mathrm{o}}$ remains unaffected by tides unless the system is at or near the point of complete destruction (as we believe is the case for BooIII). In this context, it is possible that even in the case of Sagittarius the central dispersion reflects the equilibrium value. The same studies, in particular Muñoz et al. (2008), show that when a satellite is on the brink of being completely destroyed by the action of tides, its central velocity dispersion inflates in some cases by more than a factor of 2 . If BooIII was originally a system similar to the classical dSphs, with a half-light radius of $\sim 300 \mathrm{pc}$ and a velocity dispersion of $\sim 6-7 \mathrm{~km} \mathrm{~s}^{-1}$, then its current measured value of $14 \mathrm{~km} \mathrm{~s}^{-1}$ is consistent with a scenario wherein BooIII is near complete destruction.

In order to estimate the dynamical mass of BooIII, we use a method recently developed by Wolf et al. (2009) which relies solely on the central velocity dispersion and the half-light radius of the system, and assumes neither that the density distribution of the satellite follows a King-like profile nor that mass follows light. We note that this does assume we are observing a bound system in dynamical equilibrium, which we argue is not the case for BooIII. For illustrative purposes, however, we carry out the exercise. The expression derived by Wolf et al. depends only on direct observables, and is independent of the velocity anisotropy: $M_{1 / 2}=\frac{3 \sigma_{\mathrm{LOS}}^{2} r_{1 / 2}}{G} \simeq \frac{4 \sigma_{\mathrm{LOS}}^{2} R_{\mathrm{eff}}}{G}$. Here, $\sigma_{\mathrm{LOS}}$ is the line-of-sight velocity dispersion, $r_{1 / 2}$ is the three-dimensional deprojected half-light radius, and $R_{\text {eff }}$ is the two-dimensional projected half-light radius (using their derived approximation $\left.R_{\text {eff }} \simeq(3 / 4) r_{1 / 2}\right)$. Substituting for the constants, this can be expressed as $M\left(<r_{1 / 2}\right) \simeq 9.3 \times 10^{4} \frac{R_{\text {eff }}}{100 \mathrm{pc}}\left(\frac{\sigma_{\mathrm{LOS}}}{\mathrm{km} \mathrm{s}^{-1}}\right)^{2} M_{\odot}$. Taking our measured velocity dispersion $\sigma_{\mathrm{o}}=14.0 \mathrm{~km} \mathrm{~s}^{-1}$, we find $M\left(<r_{1 / 2}\right) \simeq 1.82 \times 10^{7} \frac{R_{\text {eff }}}{100 \mathrm{pc}} M_{\odot}$, where we have left $R_{\text {eff }}$ explicit to allow for the as-yet unmeasured surface brightness profile of BooIII.

This derived mass is rather typical for a dSph systemStrigari et al. (2008) showed that Local Group dwarf galaxies all share a common mass scale of the order of $\sim 10^{7} M_{\odot}$ measured within the inner $300 \mathrm{pc}$ of these satellites (as had earlier been suggested by Mateo 1998). Note that the above mass estimate for BooIII would be three times higher if we take $R_{\text {eff }}=300 \mathrm{pc}$, although the true $R_{\text {eff }}$ is likely to be smaller (G09). Adopting $M_{\mathrm{V}}=-5.8$ gives a total luminosity of $L=1.8 \times 10^{4} L_{\odot}$. Using this value along with our mass estimate, we derive a total mass-to-light ratio of $(M / L)_{\mathrm{V}} \sim 1000 \frac{R_{\text {eff }}}{100 \mathrm{pc}}(M / L)_{V, \odot}$. A mass-to-light ratio of $\sim 1000$ is similar to those measured for the ultrafaint dSphs of similar $M_{\mathrm{V}}$ (e.g., Simon \& Geha 2007, Figure 15).

\section{DISCUSSION}

Despite the seemingly "normal" mass derived for BooIII, we argue that a number of pieces of evidence support the idea that BooIII is not in dynamical equilibrium and is likely in a transitional state between a bound system and a completely unbound stellar stream-similar to the objects described by Kroupa (1997) but with a high dark matter content. As we have already suggested, the fact that BooIII has the highest measured line-of-sight velocity dispersion of any $\mathrm{dSph}$ is consistent with the observation (from, e.g., Muñoz et al. 2008) that a satellite in the throes of complete tidal disruption can have its velocity dispersion inflated by more than a factor of 2 . Tidal disruption seems quite plausible, since the large measured Galactocentric velocity for BooIII places it in a highly elongated, and thus potentially destructive, orbit.

Morphological evidence further supports the suggestion that BooIII is a dissolving dwarf galaxy in the final stages of tidal destruction. The density distribution derived by G09 (and confirmed by Correnti et al. 2009) is quite unusual. Most dSphs, with perhaps the exception of a few of the new ultrafaint ones which are suspected to be undergoing tidal stripping, have density profiles that are well described by King-like or Plummer-like profiles. In contrast, Figure 11 of G09 (and discussion by Correnti et al. 2009) shows that BooIII is better described by a power law of index -1 . This observational result is also consistent with the idea of a satellite on the brink of destruction. Several studies (e.g., Johnston et al. 1999; Muñoz et al. 2008; Peñarrubia et al. 2008) have shown that a satellite which started with a King-like density distribution develops a power-law-like distribution in the outer parts of its luminous component as it undergoes tidal stripping (due to escaping stars at large radii). Eventually, and as the satellite becomes nearly 
unbound, the King-like core also turns into a power-law-like distribution. Furthermore, Grillmair (2009; Figure 10) showed that BooIII has irregular stellar surface density contours and is extended along the east-west direction. In Figure 4, we show the spatial distribution of our spectroscopic targets overlaid on contours of the G09 filtered star counts. Though our single $\mathrm{MMT}+$ Hectospec setup covers only a fraction of the rather large BooIII stellar overdensity, the identified RV members seem to follow the BooIII contours. The Styx stellar stream also passes through the same region of the sky where BooIII is located, and at nearly the same distance (G09). BooIII may be associated with this stream, and indeed may even be its progenitor. However, further kinematical information along the Styx stream will be necessary to confirm the association.

We thank the anonymous referee for helpful suggestions and gratefully acknowledge support by NSF grant AST-0807945 and NASA/JPL contract 1228235.

\section{REFERENCES}

Abazajian, K. N., et al. 2009, ApJS, 182, 543

Belokurov, V., et al. 2006a, ApJ, 647, L111

Belokurov, V., et al. 2006b, ApJ, 642, L137

Belokurov, V., et al. 2007, ApJ, 654, 897

Belokurov, V., et al. 2009, MNRAS, in press (http://www3.interscience.wiley. com/journal/122498764/abstract)

Bullock, J. S., \& Johnston, K. V. 2005, ApJ, 635, 931

Bullock, J. S., Kravtsov, A. V., \& Weinberg, D. H. 2001, ApJ, 548, 33

Correnti, M., Bellazzini, M., \& Ferraro, F. R. 2009, MNRAS, 397, L26

Fabricant, D., et al. 2005, PASP, 117, 1411

Friel, E. D. 1987, AJ, 93, 1388

Girardi, L., Bressan, A., Bertelli, G., \& Chiosi, C. 2000, A\&AS, 141, 371

Gorgas, J., Faber, S. M., Burstein, D., Gonzalez, J. J., Courteau, S., \& Prosser, C. 1993 , ApJS, 86, 153

Grillmair, C. J. 2006a, ApJ, 645, L37

Grillmair, C. J. 2006b, ApJ, 651, L29

Grillmair, C. J. 2009, ApJ, 693, 1118 (G09)
Grillmair, C. J., \& Dionatos, O. 2006, ApJ, 643, L17

Hargreaves, J. C., Gilmore, G., Irwin, M. J., \& Carter, D. 1994, MNRAS, 269, 957

Harris, W. E. 1996, AJ, 112, 1487

Ibata, R., Lewis, G. F., Irwin, M., Totten, E., \& Quinn, T. 2001, ApJ, 551, 294

Johnston, K. V., Sigurdsson, S., \& Hernquist, L. 1999, MNRAS, 302, 771

Kleyna, J. T., Wilkinson, M. I., Evans, N. W., Gilmore, G., \& Frayn, C. 2002, MNRAS, 330, 792

Kroupa, P. 1997, New Astron., 2, 139

Majewski, S. R., Skrutskie, M. F., Weinberg, M. D., \& Ostheimer, J. C. 2003, ApJ, 599, 1082

Martin, N. F., Ibata, R. A., Chapman, S. C., Irwin, M., \& Lewis, G. F. 2007, MNRAS, 380, 281

Mateo, M. L. 1998, ARA\&A, 36, 435

Mink, D. J., Wyatt, W. F., Caldwell, N., Conroy, M. A., Furesz, G., \& Tokarz, S. P. 2007, in ASP Conf. Ser. 376, Astronomical Data Analysis Software and Systems XVI, ed. R. A. Shaw, F. Hill, \& D. J. Bell (San Francisco, CA: ASP), 249

Muñoz, R. R., Carlin, J. L., Frinchaboy, P. M., Nidever, D. L., Majewski, S. R., \& Patterson, R. J. 2006, ApJ, 650, L51

Muñoz, R. R., Majewski, S. R., \& Johnston, K. V. 2008, ApJ, 679, 346

Oh, K. S., Lin, D. N. C., \& Aarseth, S. J. 1995, ApJ, 442, 142

Peñarrubia, J., Navarro, J. F., \& McConnachie, A. W. 2008, ApJ, 673, 226

Piatek, S., \& Pryor, C. 1995, AJ, 109, 1071

Pryor, C., \& Meylan, G. 1993, in ASP Conf. Ser. 50, Structure and Dynamics of Globular Clusters, ed. S. Djorgovski \& G. Meylan (San Francisco, CA: ASP), 357

Robin, A. C., Reylé, C., Derrière, S., \& Picaud, S. 2003, A\&A, 409, 523

Rocha-Pinto, H. J., Majewski, S. R., Skrutskie, M. F., Crane, J. D., \& Patterson, R. J. 2004, ApJ, 615, 732

Schiavon, R. P. 2007, ApJS, 171, 146

Simon, J. D., \& Geha, M. 2007, ApJ, 670, 313

Strigari, L. E., Bullock, J. S., Kaplinghat, M., Simon, J. D., Geha, M., Willman, B., \& Walker, M. G. 2008, Nature, 454, 1096

Willman, B., et al. 2005, ApJ, 626, L85

Wolf, J., Martinez, G. D., Bullock, J. S., Kaplinghat, M., Strigari, L. E., Geha, M. C., Simon, J. D., \& Willman, B. 2009, AAS Meeting Abstracts, 214, \#311.05

Worthey, G., Faber, S. M., Gonzalez, J. J., \& Burstein, D. 1994, ApJS, 94, 687

Yanny, B., et al. 2003, ApJ, 588, 824

Zucker, D. B., et al. 2006a, ApJ, 650, L41

Zucker, D. B., et al. 2006b, ApJ, 643, L103 